\title{
Non linear force feedback enhancement for cooperative robotic neurosurgery enforces virtual boundaries on cortex surface
}

\author{
Elisa Beretta ${ }^{a, *}$, Giancarlo Ferrigno ${ }^{b}$, Elena De Momi $^{b}$ \\ ${ }^{a}$ Electronics, Information and Bioengineering Department, Politecnico di Milano, p.zza Leonardo da Vinci 32, Milan, 20133, Italy, \\ E-mail: elisa.beretta@polimi.it \\ ${ }^{b}$ Electronics, Information and Bioengineering Department, Politecnico di Milano, p.zza Leonardo da Vinci 32, Milan, 20133, Italy
}

Surgeons can benefit from the cooperation with a robotic assistant during the repetitive execution of precise targeting tasks on soft tissues, such as brain cortex stimulation procedures in open-skull neurosurgery. Position-based force-to-motion control schemes may not be satisfactory solution to provide the manipulator with the high compliance desirable during guidance along wide trajectories. A new torque controller with non-linear force feedback enhancement (FFE) is presented to provide augmented haptic perception to the operator from instrument-tissue interaction. Simulation tests were performed to evaluate the system stability according to different non-linear force modulation functions (power, sigmoidal and arc tangent). The FFE controller with power modulation was experimentally validated with a pool of non-expert users using brain-mimicking gelatin phantoms (8\%-16\% concentration). Besides providing hand tremor rejection for a stable holding of the tool, the FFE controller was proven to allow for a safer tissue contact with respect to both robotic assistance without force feedback and freehand executions (50\% and $75 \%$ reduction of the indentation depth, respectively). Future work will address the evaluation of the safety features of the FFE controller with expert surgeons on a realistic brain phantom, also accounting for unpredictable tissue motions as during seizures due to cortex stimulation.

Keywords: robotic surgery; cooperative control; force feedback enhancement.

\section{Introduction}

Surgeons may benefit from the cooperation with a robotic assistant during the repetitive execution of precise targeting tasks on soft tissues, ${ }^{1,2}$ such as during brain cortex stimulation procedures in open-skull neurosurgery. ${ }^{3}$ These tasks include the smooth motion of a tool (in the working volume) and its accurate placement in a stable pose on the target tissues, thus requiring both an intuitive guidance during approaching and a safe and effective contact with tissue. Transparency, which quantifies the ability of a robot to cooperatively follow human movements without requiring any human-perceptible resistive force drive, ${ }^{4}$ is required in the target approaching phase, to reduce the fatigue of the user and the execution time. In the tool placement phase, the contact between the surgical instrument and the underneath tissue must be guaranteed stable, filtering hand tremor (physiological tremor in the band $8-12 \mathrm{~Hz}$ ), ${ }^{5}$ and safe, preventing any damage to the tissue due to indentation. Also, if predictable and/or unpredictable motions of the soft organ of interest occur, e.g. respectively due to breathing and pulsatility ${ }^{6}$ and/or to epileptic seizures during brain cortex stimulation in neurosurgery, ${ }^{7}$ the robotic assistant should be able to compensate them and react safely.

Touch interactions and physical contacts are critical fac- tors during the manipulation of tissue/objects. Haptic feedback in tele-operation and cooperative manipulation systems has been an active area of research for several decades, although its benefits in robotic surgery have not yet been assessed in terms of clinical outcome ${ }^{8}$ and major issues have prevented its use in a commercially available system ${ }^{9,10}$. In particular, the intrinsic trade-off between stability and transparency and the challenge of sensing forces under cost, biocompatibility and sterilizability constraints are still addressed. However, force feedback was proven to allow for tissue characterization, ${ }^{9,11}$ lower applied forces on tissues ${ }^{12}$ and reduced unintentional injuries. ${ }^{13}$ In particular, position-based impedance controlled (admittance) assistants, guided by the instant feedback of forces measured at the surgical instrument, prevent damage to soft tissues and reduce surgeon's fatigue both for tele-operated devices, e.g. in heart surgery, ${ }^{14}$ and for cooperative manipulation systems, e.g. in vitro-retinal surgery ${ }^{15}$ and in assisted needle insertion in keyhole neurosurgery. ${ }^{10}$ This strategy was proven very effective in highly demanding surgical procedures in terms of tool positional accuracy, thus where a "very stiff" dynamic behavior of the robotic device is desirable. Scaled force reflection strategies ${ }^{15,16}$ were developed for the Johns Hopkins' Steady-Hand Eye robot for in vitro-retinal surgery, where surgeons are required to perform micro scale maneuvers while safely applying

*p.zza Leonardo da Vinci 32, Milan, Italy 
forces that are below sensory perception. In particular, a microforce guided cooperative control, enforcing a global limit on the forces applied at the robot tool tip and actively guiding the operator towards the direction of lower resistance, proven to be effective in enforcing force limits during peeling tasks. ${ }^{15}$ Also, sclera contact location and force measurements were recently used to adapt the remote center-of-motion (RCM) constraint in a variable admittance controller in order to minimize the eye motion while enabling tool manipulation inside the eye, as well as provide useful sclera force feedback to assist the repositioning of the eye. ${ }^{17}$ Position-based force-to-motion control schema with linear force feedback enhancement were implemented also for cooperative assisted needle insertion devices, ${ }^{10}$ thus providing the user with the haptic feeling required to detect changing tissue properties of subsurface structures at different depths. All the above-mentioned force tracking control approaches exploit a manipulator with high stiffness dynamics and suffer from the inability to provide a soft compliant behavior. ${ }^{18}$

However, this approach is not advisable for surgical targeting tasks, which require the repetitive execution of wide maneuvers from/to a home robotic configuration, e.g. during brain mapping procedures. In this context, a highly compliant cooperative manipulator is desirable to promptly react to the applied forces without resisting to the surgeon guidance, reducing the fatigue of the operator and the task execution time. ${ }^{2}$ Thus, a torque-based impedance controller is more suited. ${ }^{18}$ Impedance-controlled cooperative robots, e.g. the Acrobot system with back-drivable joints for robotic assisted orthopaedic surgery, ${ }^{19}$ allow the natural transmission to the user of the interaction forces with the environment, both for human guidance and/or for tissue contact, without requiring direct force sensing. ${ }^{20}$ Nevertheless, an enhancement of the tool-tissue kinaesthetic information is needed to provide haptic sensation to the user, due to the small ranges of forces involved in the interaction with soft tissues, which may be masked by the robotic mechanical impedance, ${ }^{21}$ reducing the user perceivable level. ${ }^{22}$ In particular, interaction forces during indentation on swine brain tissue were estimated below $0.2 \mathrm{~N}$ with a $10 \mathrm{~mm}$ diameter indentor pushed at a depth of $10 \mathrm{~mm} .^{23}$ The maximum peak forces during manual and robotic direct access needle insertion respectively into in-vivo bovine liver and kidney were reported in the range $0.7-0.8$ N. ${ }^{24}$ Parameter values used to model the brain tissue with small deformation linear elastic constitutive equation vary significantly, with Young modulus between $0.6-180 \mathrm{kPa} .{ }^{25}$

In this work, we propose to cooperatively assist surgical targeting task on soft tissues with a non-linear force feedback torque control, in order to augment the surgeon skills during the placement of the surgical instrument with respect to pure visual feedback. The performance of the robotic assistance controller are assessed on brain-mimicking gelatin phantoms, which are mechanically characterized to quantitatively evaluate the tissue damage due to the contact with the tool, thus defining a safety criterion on the maximum force and penetration allowed during the indentation. The performances of the robotic assistance with and without force feedback augmentation are comparatively evaluated with respect to freehand task executions, both in terms of effectiveness in preventing the insertion of the surgical tool during the target approaching and of position/force tremor rejection during the holding phase.

The paper is organized as follows: the proposed enhanced force feedback loop is presented in Section II, together with the stability analysis and the experimental performance evaluation on brain-mimicking phantoms; simulation and experimental results are presented in Section III and discussed in Section IV.

\section{Material and Methods}

\subsection{Torque-based impedance control}

A torque-based impedance control for flexible joint manipulators $^{20}$ is used to provide a soft dynamic behavior of the robotic arm during the assisted guidance. As shown in Fig. 1, the torque input of the joint state feedback controller $(\tau)$ is computed as:

$$
\tau=\tau_{d}+\tau_{e}-\hat{\tau}_{n}
$$

where $\tau_{d}$ is the desired torque vector, $\hat{\tau}_{n}$ is the feed forward model-based torque vector for the compensation of the robot dynamics, i.e. gravity and Coriolis-centrifugal terms, and $\tau_{e}$ is the vector of the external torques measured by the joint torque sensors of the manipulator. Two different types of environmental interaction $\left(\mathbf{f}_{e}\right)$ are considered in this work:

- Human interaction forces $\left(\mathbf{f}_{h}\right)$ : the operator guides the manipulator applying external forces with a single-point contact during the free-motion guidance phase and the instrument placement phase, depending on his/her unknown intention of motion; ${ }^{26}$

- Soft tissue interaction forces $\left(\mathbf{f}_{t}\right)$ : the compression /indentation reaction of the soft tissue during the instrument placement phase depends on its viscoelastic mechanical properties. $^{27}$

Thus, the external torque vector can be written as:

$$
\tau_{e}=J^{T}\left(\mathbf{f}_{h}+\mathbf{f}_{t}\right)+\tau_{n}
$$

where $J$ is the Jacobian matrix of the manipulator and $\tau_{n}$ represents the real effects of the robot dynamics. Non-idealities of the robot dynamics model $\left(\hat{\tau}_{n} \neq \tau_{n}\right)$ limit the maximum transparency of the system. ${ }^{26}$ In fact, during the target placement phase, which can be assumed as a quasi-static scenario, the residual friction of the manipulator may mask the delicate interaction forces generated by the contact with the soft tissue. Thus, a force feedback enhancement loop is required to provide haptic perception to the user during tissue indentation.

\subsection{Force Feedback enhancement}

The robotic system is provided with a force sensor placed between the flange and the instrument to allow the direct measurements of the soft tissue interaction forces $\left(\mathbf{f}_{t}\right)$. Considering a generic augmentation function $\left(\mathbf{f}_{t}^{\prime}=g\left(\mathbf{f}_{t}\right)\right)$, the enhanced forces are fed back in the control loop as desired torque vector:

$$
\tau_{d}=J^{T} \mathbf{f}_{t}^{\prime}
$$




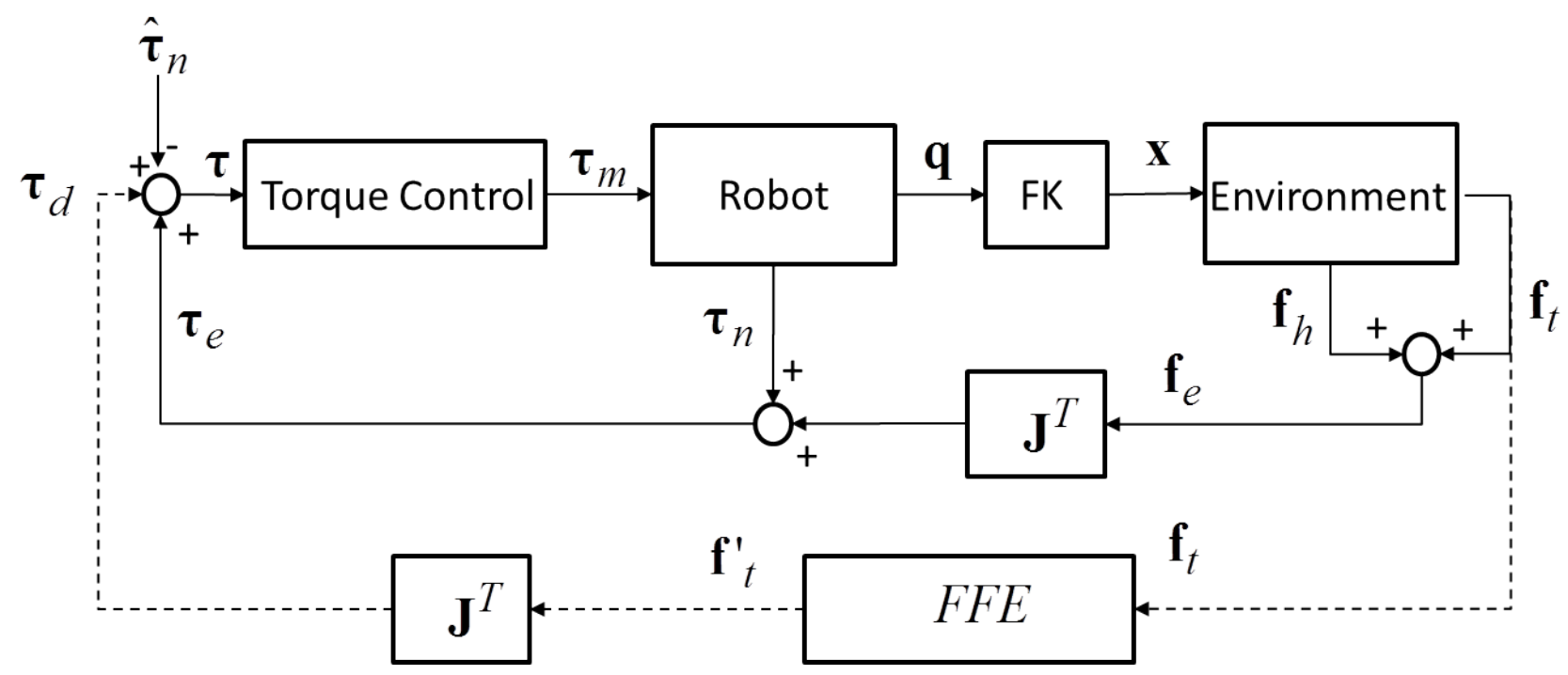

Fig. 1. Torque control with force-feedback enhancement.

Thus, the balance of forces at the contact point $(\tau=0)$, assuming $\hat{\tau}_{n}=\tau_{n}$, results:

$$
\begin{gathered}
J^{T}\left(\mathbf{f}_{t}^{\prime}+\mathbf{f}_{t}+\mathbf{f}_{h}\right)=0 \\
\mathbf{f}_{h}=-\left(\mathbf{f}_{t}^{\prime}+\mathbf{f}_{t}\right) \approx-\mathbf{f}_{t}^{\prime}
\end{gathered}
$$

considering the tissue interaction force negligible with respect to the augmented force. Different force feedback enhancement approaches are considered to provide augmented haptic perception. In particular, non-linear force modulations are investigated to provide variable force resolution and smoothly apply force saturation. The following common non-linear modulations $\left(\mathbf{f}_{t}^{\prime}=g\left(\mathbf{f}_{t}\right)\right)$ were implemented and compared:

(i) Power function with saturation (pow):

$$
\mathbf{f}_{t}^{\prime}=\left\{\begin{array}{cc}
0 & \left\|\mathbf{f}_{t}\right\| \leq \mathbf{f}_{t} \\
\mathbf{f}_{s} & \left\|\mathbf{f}_{t}^{\prime}\right\|>\mathbf{f}_{s} \\
\rho\left(\mathbf{f}_{t}\right)^{n} & \text { elsewhere }
\end{array}\right.
$$

where $\rho$ is a positive scalar $(\rho>1), n$ is the positive exponent of the polynomial function in the range (0-1], $\mathbf{f}_{s}$ is a fixed positive saturation level and $\mathbf{f}_{t}$ is the minimum force threshold accounting for the sensor measurement error. The degree of non linearity and the curve slope are decoupled from the range of forces defined by the saturation level, allowing for 3 tuning degrees-of-freedom (dof). Despite the continuous force modulations, their derivatives present two discontinuities at the minimum force threshold and at the saturation point, respectively.

(ii) Sigmoidal function ( $\mathrm{sig}$ ):

$$
\mathbf{f}_{t}^{\prime}=\frac{2 \mathbf{f}_{s}}{1+e^{-\alpha \mathbf{f}_{t}}}-\mathbf{f}_{s}
$$

where $\alpha$ and $\pm \mathbf{f}_{s}$ represent the steepness and the horizontal asymptotes of the curve respectively ( 2 tuning dof). The curve derivative in the origin $\left(\left.\frac{d \mathbf{f}_{t}^{\prime}}{d \mathbf{f}_{t}}\right|_{\mathbf{f}_{t}=0}\right)$ is finite and equal to $\frac{\alpha}{2} \mathbf{f}_{s}$. However, force resolution profile and saturation are not independent.

(iii) Arc tangent function (atg):

$$
\mathbf{f}_{t}^{\prime}=\frac{2 \mathbf{f}_{s}}{\pi} \arctan \left(\beta \mathbf{f}_{t}\right)
$$

where $\beta$ and $\pm \mathbf{f}_{s}$ represent the steepness and the horizontal asymptotes of the curve respectively ( 2 tuning dof). The curve derivative in the origin is finite and equal to $\frac{\beta}{\pi} 2 \mathbf{f}_{s}$. However, force resolution profile and saturation are not independent.

For all approaches, the saturation level $\left(\mathbf{f}_{s}\right)$ defines the maximum provided force feedback, thus representing the maximum force the user has to overcome to intentionally penetrate the tissue. In order to comparatively evaluate the above-mentioned enhancement approaches, the tuning of the function parameters was performed based on the following criteria:

- Maximum enhancement constraint, which provide the value of the force saturation $\left(\mathbf{f}_{s}\right)$;

- Minimum enhancement constraint, which defines the minimal enhancement level required to provide the user with a feedback higher than the perceivable force $\left(\underline{\mathbf{f}}_{h}\right)$ before the puncturing point of the tissue $\left(\overline{\mathbf{f}}_{t}\right)$, guaranteeing a safe contact and indentation.

Fig. 2 shows the three different force enhancement approaches and their parameters.

\subsection{Simulation analysis for stability}

In order to perform the stability analysis, the overall system was divided in four blocks, as shown in Fig. 3. The torque control 
for flexible manipulator herein presented was proven to be passive without force feedback enhancement ${ }^{20}$ when in contact with passive environment.

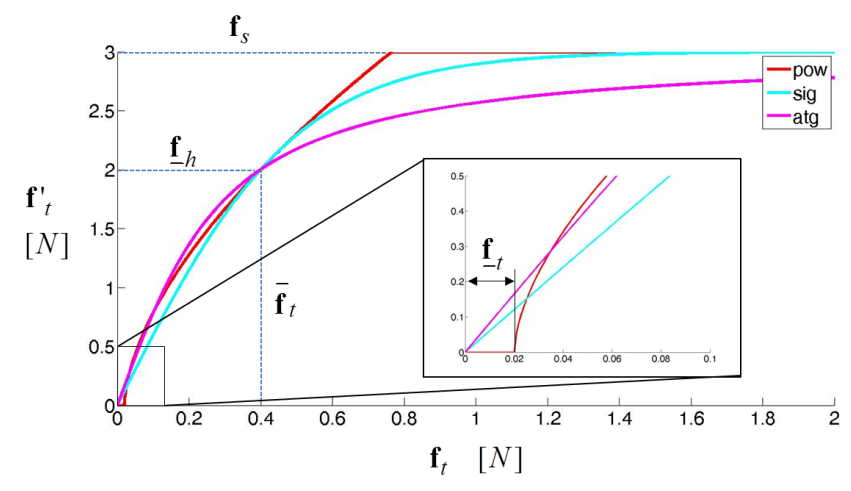

Fig. 2. Comparison of the Force Feedback enhancement modulation approaches: power with saturation (pow), sigmoidal (sig) and arc tangent $(a t g)$. The common tuning parameters, i.e. force saturation $\left(\mathbf{f}_{s}\right)$, force threshold $\left(\underline{\mathbf{f}}_{t}\right)$ and minimum enhancement constraint $\left(\overline{\mathbf{f}}_{t}, \underline{\mathbf{f}}_{h}\right)$, are also shown. For $\mathbf{f}_{t}<0$ the plot is odd symmetric.

Assuming the soft tissue to be linear and isotropic, ${ }^{25}$ its dynamic behavior is modeled as a II order Mass $(m)$-Spring $(k)$ Damper(b) (MSD) system as:

$$
f=(m \ddot{x}+b \dot{x}+k x)
$$

where $x=0$ represents the equilibrium point on the tissue surface, where null tissue compression/extension occurs. This system is proven to be passive using the semi-positive Lyapunov function $(L)$ representing the total system energy storage (potential and elastic) as:

$$
L=\frac{1}{2} k x^{2}+\frac{1}{2} m \dot{x}^{2}
$$

The system power is computed as:

$$
\dot{L}=k x \dot{x}+m \dot{x} \ddot{x}=\dot{x}(f-b \dot{x})
$$

from which $\dot{L}<f \dot{x}$ is always verified for $b>0$. As well, the passivity of the feedback loop with linear enhancement can be demonstrated using the same approach. The stability features of the different non-linear force enhancement controllers were evaluated in simulation using the Matlab-Simulink (sampling frequency $1 \mathrm{KHz}$ ). In particular, the tissue MSD model was tuned in order to simulate the indentation behavior of brain tissue $(m=0.1 \mathrm{~N}, b=10 \mathrm{Ns} / \mathrm{m}, k=80 \mathrm{~N} / \mathrm{m})$, considering a critical puncturing scenario with contact forces $<0.5 \mathrm{~N}$ for indentation level $<5 \mathrm{~mm}$ using a $3 \mathrm{~mm}$ diameter indentor. These values were confirmed by the experimental characterization protocol performed on the brain-mimicking gelatin phantoms (see 2.4.1).

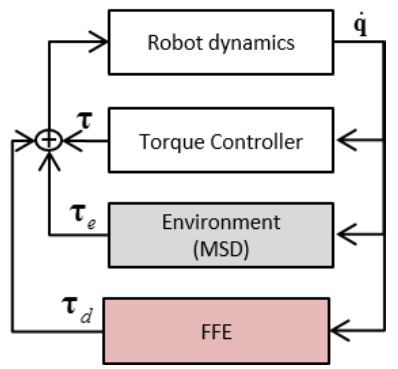

Fig. 3. Block scheme of the system for the stability analysis, where $\dot{\mathbf{q}}$ represents the joint velocities.

The force measurement error $(e)$ was modeled using an additive Gaussian noise $(N(0, \sigma))$ with null mean and standard deviation $\sigma$. The noisy force output of the tissue model is then enhanced with the three force enhancement modulations, i.e. power, sigmoidal and arc tangent. The stability of the modulation was evaluated through the computation of the spectrum of the enhanced force signals $(Y(f))$, in order to compare the power distribution on the fundamental frequency and harmonics. The system was excited by sinusoidal position inputs at different amplitudes $(1-7 \mathrm{~mm})$ and frequency $(0.1-10 \mathrm{~Hz})$, which span from an indentation gesture $(0.1 \mathrm{~Hz})$ to the mean physiological hand tremor $(10 \mathrm{~Hz}){ }^{5}$ A simplified simulation example is shown in Fig. 4: the force outputs of the three non-linear enhancement methods are reported over time and over frequency together with the sinusoidal force signal (amplitude $=1 \mathrm{~N}$, frequency $=5 \mathrm{~Hz}$, null measurement noise) simulated as the output of the MSD block. The harmonic distortion index $(D)$ was computed for each simulated input condition as:

$$
D=\sqrt{\sum_{i=2}^{N} \frac{x_{i}^{2}}{x_{1}^{2}}} \cdot 100
$$

where $x_{1}$ is the amplitude of the fundamental frequency and $i$ is the index running on the harmonics until the $N$-th order. Also, an enhancement gain index was computed as the ratio between the spectrum modules of the fundamental frequency of the enhancement method and of the force signal output of the MSD model (f). These indexes give an estimate of the difference between the non-linear behaviors and the linear one, which was demonstrated passive.

\subsection{Experimental Protocol}

The proposed control approach was experimentally evaluated using the LWR4+ (Kuka, Augsburg, Germany), 7 degreesof-freedom (DoFs) flexible joint manipulator, which features $0.05 \mathrm{~mm}$ repeatability (as per datasheet) and $1 \mathrm{~mm}$ accuracy. ${ }^{28}$ Joint torque sensors allow the measurements of external torques along the manipulator structure $\left(\tau_{e}\right)$. In order to distinguish between the interaction forces due to the human guidance and to 

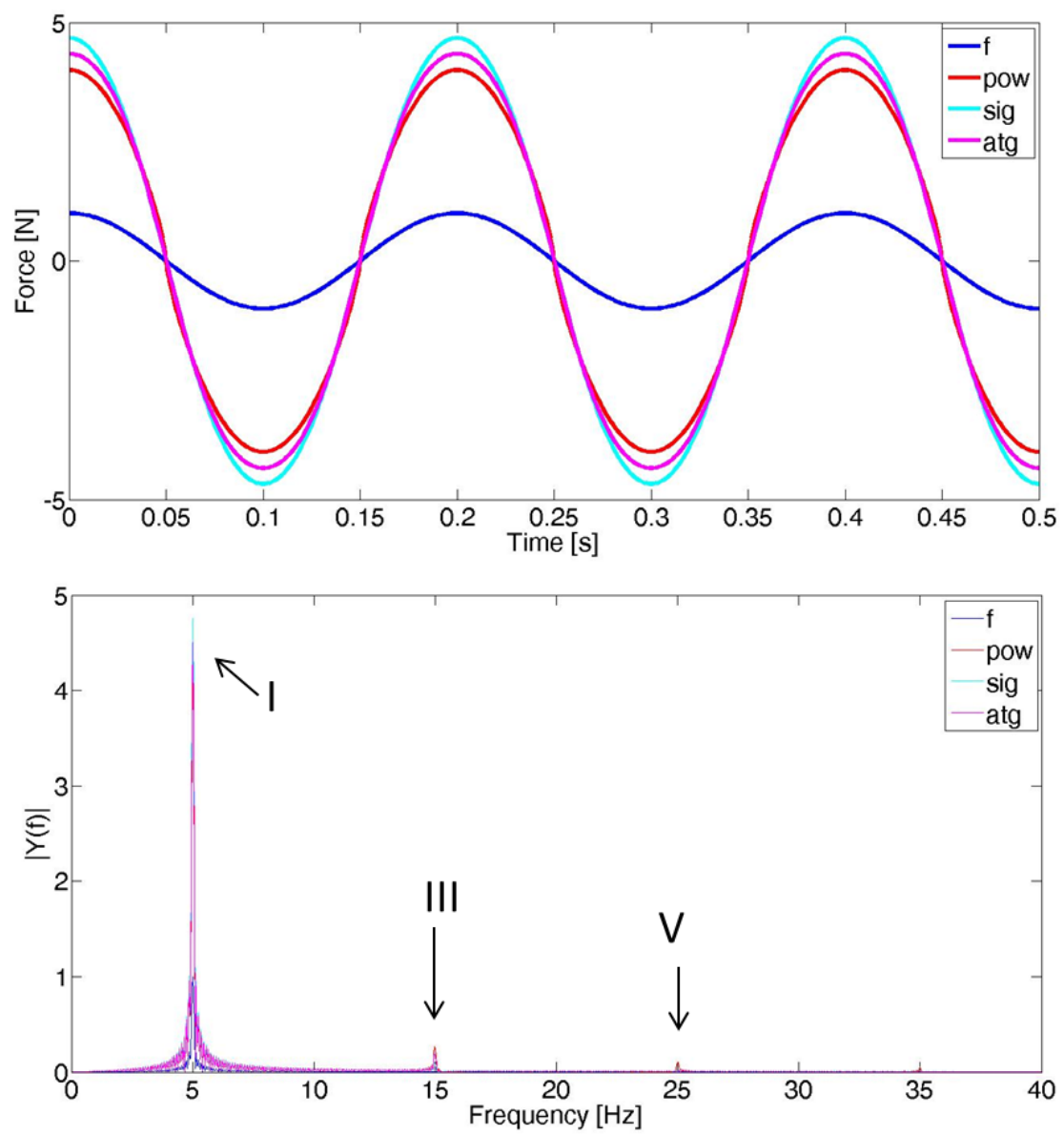

Fig. 4. Example of a simulation results for a sinusoidal wave with $1 \mathrm{~N}$ amplitude and $5 \mathrm{~Hz}$ frequency without measurement noise. Comparison in time (top) and spectrum module (bottom) of the outputs of the three non-linear force enhancement approaches (pow, sig and atg) together with the input signal (f). The I, III and V harmonic components of the spectrum are also identified.

the soft tissue indentation, a 6 DoFs Nano43 force/torque (F/T) sensor (ATI Industrial Automation, Apex, NC), which features $1 / 512 \mathrm{~N}-1 / 40 \mathrm{Nmm}$ resolution in a $9 \mathrm{~N}-125 \mathrm{Nmm}$ range, was mounted between the robot flange and a straight tool (3mm diameter tip), mimicking the coaxial surgical tool for brain stimulation (Fig. 5). In order to compare free-hand and robotic assisted task executions, the Gamma F/T sensor (ATI Industrial Automation, Apex, NC), which features $1 / 80 \mathrm{~N}-1 / 2 \mathrm{Nmm}$ resolution in a $32 \mathrm{~N}-2.5 \mathrm{Nm}$ range, was also used as a load cell to measure the interaction forces on the soft material phantoms. A 3D-printed horizontal calibration plate with two orthogonal axes $\left(R F_{P}\right)$ was rigidly fixed to the load cell. As shown in Fig. 5, the Certus optical localization system (Northern Digital Instruments, Ontario, Canada) with active markers ( $0.1 \mathrm{~mm}$ accuracy) was used to track the instrument pose $\left(R F_{E E}\right)$ and the calibration plate $\left(R F_{C P}\right)$ in the camera frame $\left(R F_{C}\right)$. A reference frame with the origin coincident with the tip of the tool and the $\mathrm{z}$-axis coaxial with the tool's principal axis $\left(R F_{T C P}\right)$ was defined with respect to the end effector reference frame $\left(R F_{E E}\right)$ using a piv- oting procedure. Using a hand-eye calibration procedure, ${ }^{29}$ the tool tip position $\left(t_{P}\right)$ and the force measurements of the endeffector sensor $\left(f s_{P}\right)$ and of the load cell $\left(l c_{P}\right)$ were computed in $R F_{P}$ (using the CAD models of the printed plate).

Tests were performed mimicking the target approaching gestures of an open-skull neurosurgical procedure using gelatin phantoms with three different mass concentrations $(8,12,16$ $\%)$, that mimic the viscoelastic characteristics of the brain tissue. ${ }^{30}$ The flat surface of each phantom was calibrated in the robot base reference frame with a least square regression process on 12 points acquired with an optical pointer (root mean square error $<0.5 \mathrm{~mm}$ ). The robotic control system was implemented in the OROCOS and ROS frameworks (www.orocos.org, www.ros.org) and controlled in a real-time environment by a Xenomai patched kernel (www.xenomai.org). Torque commands were computed at $1 \mathrm{kHz},{ }^{20}$ while the F/T signals were acquired at $2 \mathrm{kHz}$. The end-effector F/T measurements were processed with a notch filter (cut frequency $186.5 \mathrm{~Hz}$, attenuation $-50 \mathrm{~dB}$ ) to cancel out the noise derived from the robot 
fans vibration. Also, the dynamics of the linear tool was online compensated, ${ }^{31}$ resulting in a $0.02 \mathrm{~N}$ accuracy of the force measurements at the end-effector.

\subsubsection{Tissue characterization and FFE tuning}

During brain stimulation the surgeon places the stimulators in contact with the brain cortex. Large levels of indentation, up to possible penetration, may cause tissue damage. In order to quantitatively define in which range of forces penetration occurs, thus identifying a safety criterion for the gelatin indentation, the gelatin phantoms were characterized in terms of puncturing force $\left(\overline{\mathbf{f}}_{t}\right)$ and penetration level $\left(\overline{\mathbf{p}}_{t}\right)$. The protocol included 8 trials of autonomous robotic tool insertions at four different velocities $(4-16 \mathrm{~mm} / \mathrm{s})$ performed for each gelatin at different concentrations $(8-16 \%)$. Each gelatin phantom was kept for at least 8 hours at a temperature of $5 \mathrm{C}$ before use and changed at every experiment to avoid degradation of the material due to the environmental temperature $\left(25^{\circ} \mathrm{C}\right)$. The $\mathrm{F} / \mathrm{T}$ signals from the end-effector sensor $\left(f_{P}\right)$ were processed with a first order adaptive filter ${ }^{32}$ to identify the peak related to the puncturing event. The median value and the first and third Inter Quartile Ranges (IQR 25\%; IQR 75\%) of the puncturing forces and the corresponding penetration level among trials are reported in Table 1. A safety level for each different gelatin concentration is identified according to the minimum puncturing force $(0.4 \mathrm{~N}$ with $8 \%$, $0.8 \mathrm{~N}$ with $12 \%, 1 \mathrm{~N}$ with $16 \%$ ). The force feedback enhancement parameters, both for simulation and for experimental testing, were optimized considering the critical tool insertion scenario ( $8 \%$ gelatin at $4 \mathrm{~mm} / \mathrm{s}$ ), which reports the minimal puncturing force (mean value $0.41 \mathrm{~N}$ at $\approx 5.5 \mathrm{~mm}$ mean penetration) (Table 1). Due to the residual static friction of the system $( \pm 1.5 \mathrm{Nm}$ internal thresholds on the robot joint torque sensors), the minimum user perceivable force $\left(\mathbf{f}_{h}\right)$ during assisted targeting tasks is strictly related to the static friction of the system $(\approx 2 / 3 N)$ and was considered equal to $4 N$.

\subsubsection{Experimental performance evaluation}

The effectiveness of the proposed FFE controller was experimentally evaluated during repetitive targeting tasks on a pool of 6 non-expert users. The targeting task was defined as approaching an arbitrary point of the gelatin surface and holding the pose for $2 \mathrm{~s}$, mimicking the basic gesture performed during the cortex electrical stimulation. The users were asked to keep contact with the tissue during the interaction, minimizing gelatin indentation and preventing unwanted gelatin damage. Every user performed 8 targeting gestures in a randomized order on $8 \%, 12 \%$ and $16 \%$ gelatin samples in these modalities:

- Freehand (FH): the targeting task was performed with an optical pointer ( $3 \mathrm{~mm}$ diameter tip); the pencil-style hand grip is equal to the standard bipolar stimulator tools;

- Robotic assistance (RA) without force feedback;

- Robotic assistance with force feedback enhancement loop (FFE).

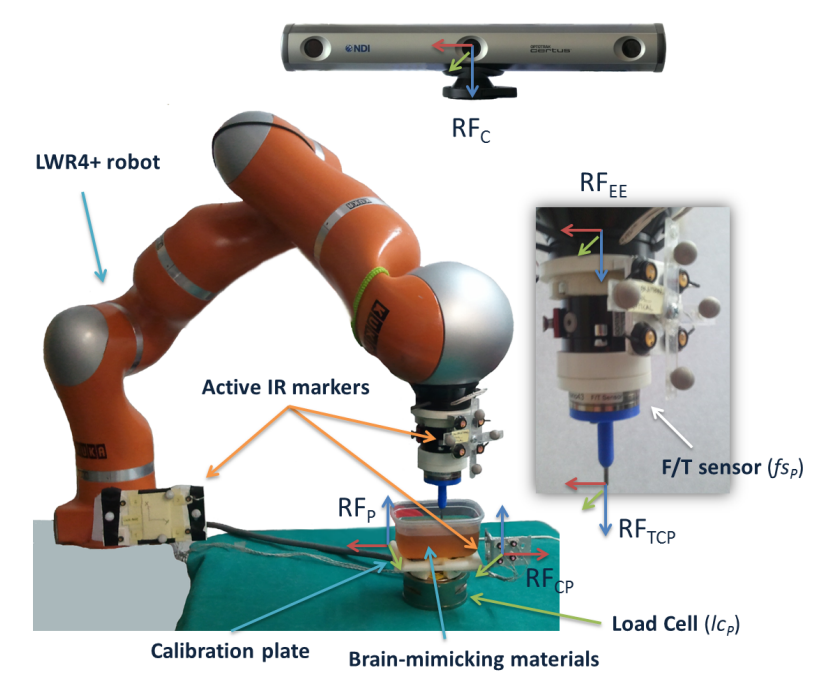

Fig. 5. The experimental setup. The reference frames (RFs) of interest are also reported.

Table 1. Results of the autonomous tool insertion protocol: (*) critical tool insertion scenario reporting the minimum puncturing force.

\begin{tabular}{lcccc}
\hline $\mathbf{f}_{t}[\mathrm{~N}]$ & $4[\mathrm{~mm} / \mathrm{s}]$ & $8[\mathrm{~mm} / \mathrm{s}]$ & $12[\mathrm{~mm} / \mathrm{s}]$ & $16[\mathrm{~mm} / \mathrm{s}]$ \\
\hline $8 \%$ & $0.41 \pm 0.02 *$ & $0.50 \pm 0.02$ & $0.52 \pm 0.02$ & $0.57 \pm 0.03$ \\
$12 \%$ & $0.78 \pm 0.02$ & $0.96 \pm 0.03$ & $1.08 \pm 0.02$ & $1.14 \pm 0.05$ \\
$16 \%$ & $1.04 \pm 0.03$ & $1.27 \pm 0.05$ & $1.42 \pm 0.03$ & $1.44 \pm 0.04$ \\
\hline$\overline{\mathbf{p}}_{t}[\mathrm{~mm}]$ & $4[\mathrm{~mm} / \mathrm{s}]$ & $8[\mathrm{~mm} / \mathrm{s}]$ & $12[\mathrm{~mm} / \mathrm{s}]$ & $16[\mathrm{~mm} / \mathrm{s}]$ \\
\hline $8 \%$ & $5.5 \pm 0.3 *$ & $6.3 \pm 0.4$ & $6.4 \pm 0.2$ & $6.3 \pm 0.4$ \\
$12 \%$ & $5.3 \pm 0.1$ & $6.1 \pm 0.2$ & $6.4 \pm 0.1$ & $6.5 \pm 0.4$ \\
$16 \%$ & $5.5 \pm 0.2$ & $6.4 \pm 0.3$ & $6.7 \pm 0.2$ & $6.7 \pm 0.1$ \\
\hline
\end{tabular}

In both the robotic assisted cases (RA and FFE), the user performed the targeting tasks guiding the 3 translational DoFs of the manipulator, while the orientation of the tool was fixed to an initial configuration. During each trial, the force and TCP position signals were processed, in order to detect the beginning/end of the holding phase during the tool-tissue contact. The following indexes of performance were evaluated for each trial:

- Mean equilibrium force $\left(F_{H}\right)$ : computed as the norm of the time average of the force signal from the load cell measurements $\left(l c_{P}\right)$;

- Mean penetration level $\left(P_{H}\right)$ : computed as the time average of the signed distance from the registered surface $(p)$, where positive values refers to position below the surface, i.e. indentation depths;

- Penetration variability $(V)$ : computed as the root mean square (RMS) distance from the mean penetration level $P_{H}$. 
Due to the significant surface registration error $(0.5 \mathrm{~mm})$, the penetration measurements of each gelatin phantom was corrected with an offset level, computed as the mean of the penetration levels measured at the instant in which the synchronized force signals overcomes its noise threshold $(0.02 N)$ for a time window larger than 200ms. An exemplary trial execution for each control modality on $16 \%$ concentration gelatin phantom is reported in Fig. 6. The median value and the first and third IQR were computed over different trials and different users for $F_{H}$ and $P_{H}$ indexes. A comparative analysis of the different task execution modalities (FH, RA, FFE) and of the different tissue mechanical properties (8-16\% gelatin concentration) was carried out using respectively the Wilcoxon signed-rank test $(p<0.05)$ and Kruskal-Wallis test $(p<0.05)$, both with Bonferroni-Holms correction $^{33}$ for multiple groups. The penetration variability index $V$ was computed over trials of each user in each execution modality (FH, RA, FFE) and for each gelatin concentration $(8 \%, 12 \%, 16 \%)$ in order to evaluate if the variability in freehand modality was greater than in the robotic assisted cases. The amplitude variability indexes among users were also computed.

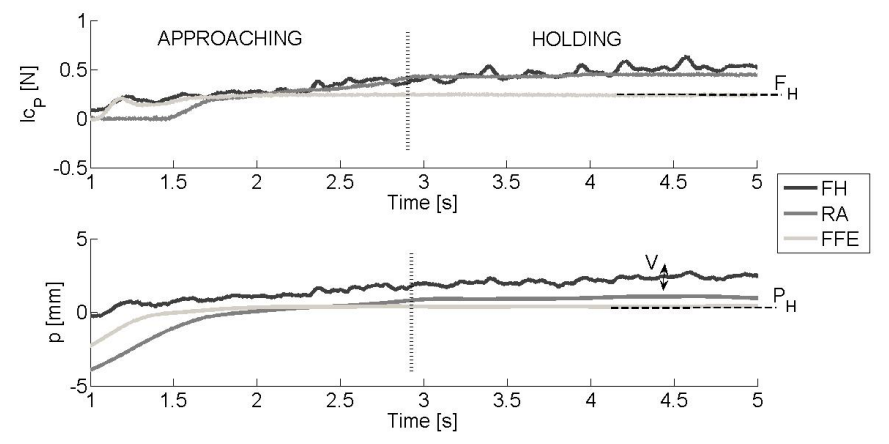

Fig. 6. Exemplary force $\left(l c_{P}\right)$ and penetration $(p)$ signals measured during one targeting gesture performed by a user on $16 \%$ gelatin phantom for each execution modality (FH, RA, FFE). The performance indexes are also reported.

\section{Results}

\subsection{Simulation}

The harmonic distortion index $D$ computed on the output of the three proposed force enhancement methods is reported in Fig. 7 with respect to different amplitude and frequency of the sinusoidal position inputs. It has to be noted that the system transfer function is odd, thus only the odd harmonics are excited. As expected, the distortion effects affect all three methods. Its value with sig and atg methods increase proportionally to both amplitude and frequency of the input signal, showing a semi-linear behavior for tissue indentation greater than $4 \mathrm{~mm}$ (reaching $0.6 \%$ and $2.5 \%$ distortion respectively at $7 \mathrm{~mm}$ indentation). On the contrary, the pow modulation presents a constant distortion effect $(<1.3 \%)$ for the whole simulated indentation range, resulting in the highest distortion for small indentations $(<5 \mathrm{~mm})$, due to the presence of derivative discontinuity close to the origin. However, the signal distortion, that potentially could affect the system's stability, is significantly limited for all three methods $(<1.5 \%)$ when a realistic range of displacement is considered for tissue indentation below the puncturing range $(<5 \mathrm{~mm})$. The enhancement gain computed for each method with respect to the input spectrum module (f) is reported in Fig. 8. The pow modulation provides greater gain for indentation below the puncturing level $(5 \mathrm{~mm})$, with enhancement factors $(>50 \%$ and $>30 \%)$ compared to sig and atg methods respectively. In conclusion, the control system is BIBO (Bounded Input - Bounded Output) stable.

\subsection{Experiments}

The results of the comparative analysis performed on the mean equilibrium force $\left(F_{H}\right)$ and penetration $\left(P_{H}\right)$ indexes during the holding phase are reported in Fig. 9. The mean equilibrium forces $F_{H}$ resulted significantly smaller when the task was executed in FFE mode (75\% quartile value below the $0.2 \mathrm{~N}$ perception threshold) with respect to both FH and RA modes with all the gelatin phantoms. While the comparable equilibrium forces computed in FH and RA modes significantly increased according to higher gelatin concentrations, no statistically significant difference was found between the $12 \%$ and $16 \%$ gelatin phantoms in FFE mode (mean value $<0.1 N$ ). The $F_{H}$ indexes resulted below the safety level identified for the different gelatin concentration $(0.4 \mathrm{~N}$ with $8 \%, 0.7 \mathrm{~N}$ with $12 \%, 1 \mathrm{~N}$ with $16 \%)$. Accordingly, the penetration depth $\left(P_{H}\right)$ during FH task executions (mean value $>2 \mathrm{~mm}$ ) resulted significantly greater with respect to RA (mean depth in the range $(1.3-1.7) \mathrm{mm}$ ) and FFE (mean depth $<1.2 \mathrm{~mm}$ ) modes. No statistically significant differences were found among the penetration depths on gelatin phantoms with different concentrations both for the FH (except for the comparison between $8 \%$ and $12 \%$ gelatins) and RA modes. Conversely, the penetration depths on $16 \%$ gelatin during FFE task executions resulted significantly smaller than the other cases, with a mean penetration level comparable to the surface registration accuracy $(0.5 \mathrm{~mm})$. As reported in Tab. 2 , the amplitude variability computed among users on the penetration depth $(V)$ signals during the holding phases in the assisted task executions (RMS error $<0.1 \mathrm{~mm}$ ) resulted significantly reduced with respect to the freehand modality (RMS error $>0.6 \mathrm{~mm}$ ).

Table 2. Penetration variability results: $(*)$ the assumption $\left(V_{F H}>V_{R A}\right)$ and $\left(V_{F H}>V_{F F E}\right)$ is verified among trials and users.

\begin{tabular}{lccc}
\hline$V[\mathrm{~mm}]$ & $8 \%$ & $12 \%$ & $16 \%$ \\
\hline FH & $0.8^{*}$ & $0.6^{*}$ & $0.6^{*}$ \\
RA-FFE & $<0.1$ & $<0.1$ & $<0.1$ \\
\hline
\end{tabular}

\section{Discussion}

In order to enhance the human-robot cooperation during targeting tasks on soft tissues in surgery, such as brain cortex stimula- 
October 3, 2015 9:40 manuscript review

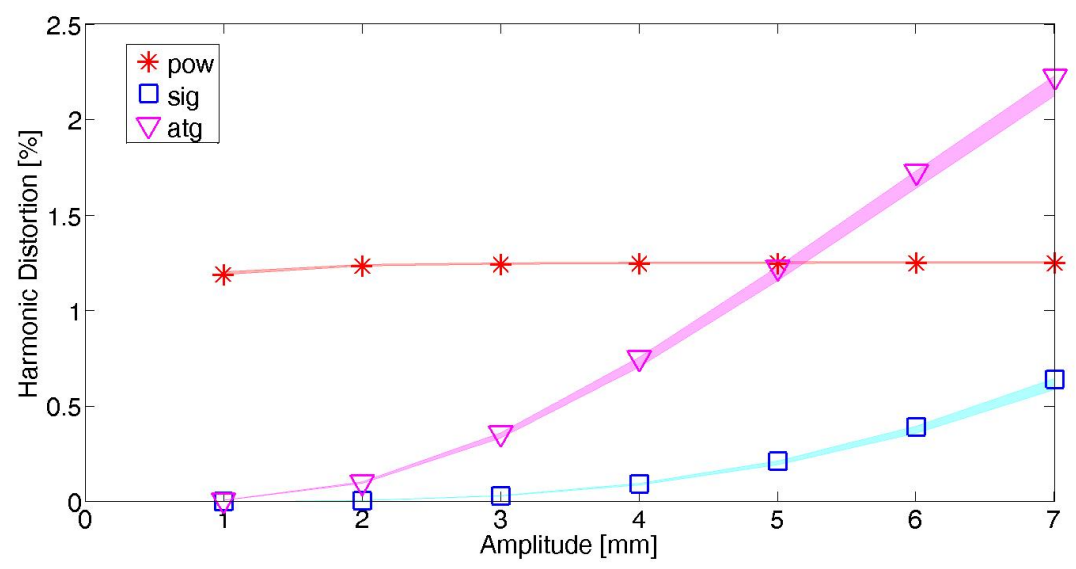

Fig. 7. Simulation results. The distortion of the three force enhancement methods (pow, sig and atg) is represented with respect to different amplitude $((1-7) \mathrm{mm})$ of the sinusoidal position input signal with the addition of zero mean Gaussian noise $(\sigma=0.02 \mathrm{~N})$. The distortion results of different input frequencies $((0.1-10) \mathrm{Hz})$ are represented by the upper and lower shadow limitation for each method.

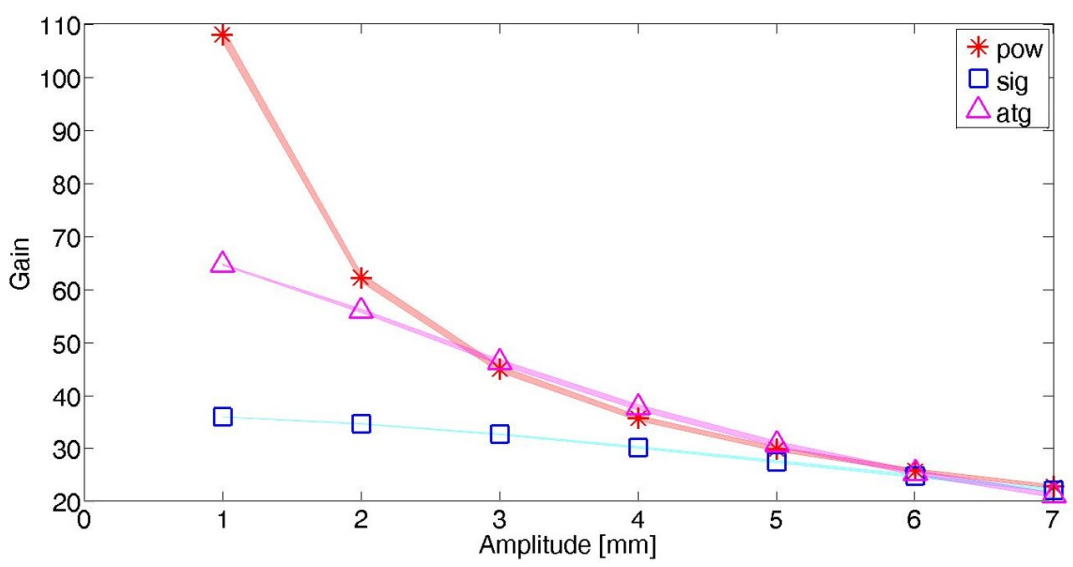

Fig. 8. Simulation results. The gains of the three force enhancement methods are represented with respect to different amplitude $((1-7) m m)$ of the sinusoidal position input signal with the addition of zero mean Gaussian noise $(\sigma=0.02 N)$. The gain results of different input frequencies $((0.1-10) H z)$ are represented by the upper and lower shadow limitation for each method.

tion procedures in open-skull neurosurgery, a torque controller with non-linear force feedback (FFE) is proposed. The controller provides enhanced haptic perception to the operator when the surgical tool is in contact with the soft tissue. In fact, such contact forces between the surgical tool and the soft tissues, e.g. brain $^{23}$ (peak forces below $0.2 \mathrm{~N}$ ) and/or liver ${ }^{24}$ (peak forces below $1 \mathrm{~N}$ ), are too small to be perceived by the user during the robotic guidance, ${ }^{26}$ due to the residual friction of the manipulator. Differently from vitro-retinal surgery ${ }^{15}$ and/or needle insertion procedures, ${ }^{10}$ in which the accuracy requirements are very high in a constrained and limited working space, position-based force-to-motion control schemes ${ }^{17}$ may not be the best suitable solution to provide the manipulator with the high compliance desirable during wide motion targeting tasks, e.g. brain cortex stimulation. The proposed approach is based on a torque-based cooperative control with a force feedback augmentation strategy, to augment the surgeon ability in performing a safe and stable contact with the soft tissue.

The stability analysis of the system was performed in simulation with three different non-linear force enhancement profiles. Although it was confirmed to be the critical function in terms of spectral distribution due to its derivative discontinuities, the pow modulation, i.e. power enhancement with saturation, proved acceptable signal distortion $(<1.5 \%$ for indentation below the puncturing tissue level, $5 \mathrm{~mm}$ ), when modeling contact with brain soft tissue $(80 \mathrm{~N} / \mathrm{m}$ stiffness) and limited measurement noise $(\sigma=0.02 N)$. Since it provides the highest number of degrees-of-freedom for specific tuning and the greatest enhancement gain for small indentations, the pow modulation was implemented in the FFE controller and evaluated in the experimental session. Also, the experimental proof of pow stability would insure the stability property of the other modulation func- 

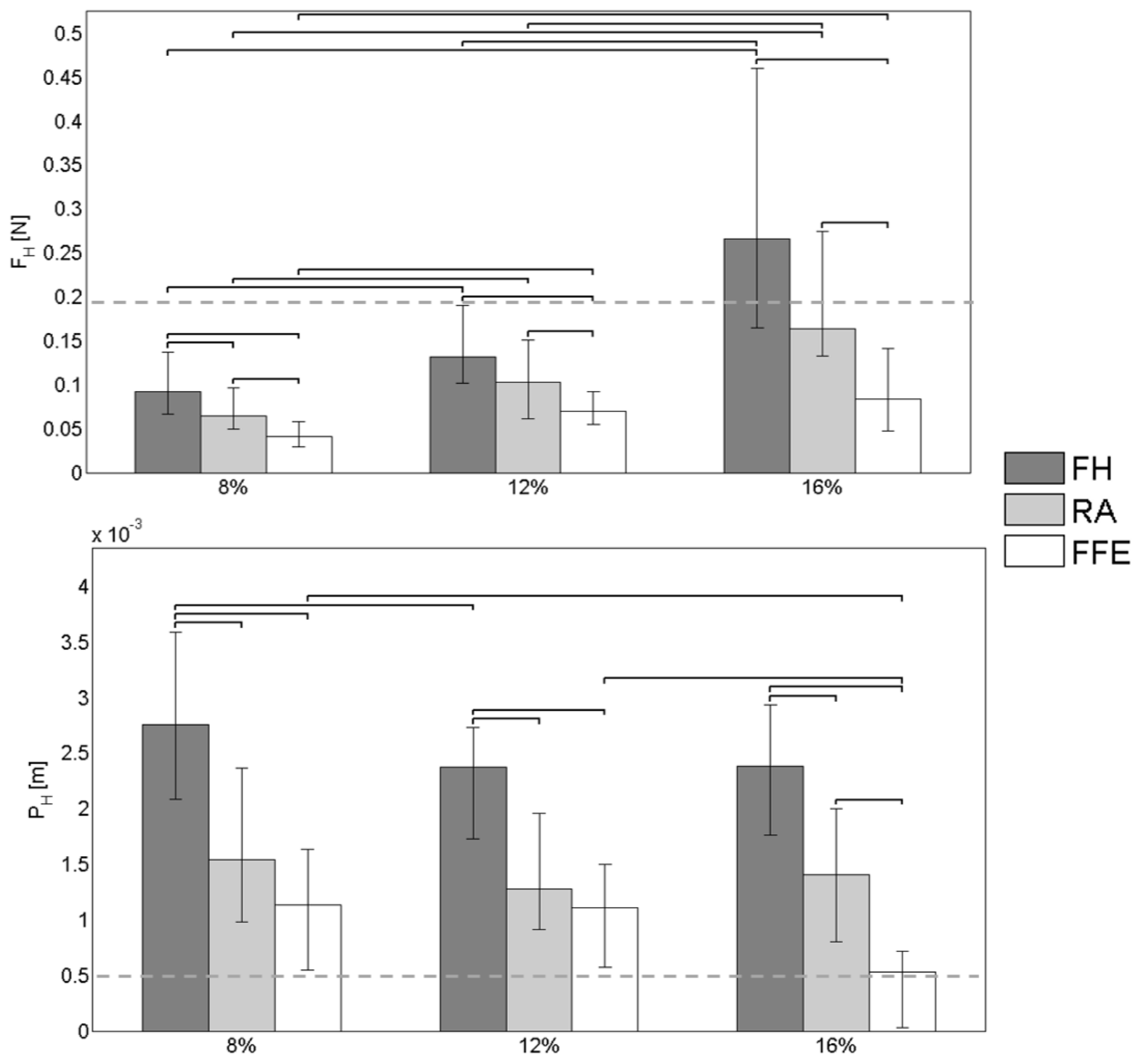

Fig. 9. Evaluation of the equilibrium force $F_{H}$ and penetration $P_{H}$ indexes using the three task execution modalities (FH, RA, FFE) and the three different concentration gelatin phantoms $(8 \%, 12 \%, 16 \%)$, for all trials of the 6 non-expert users. Vertical bars represent median values and quartiles $(25 \%$ and $75 \%)$ of performance index population. Horizontal lines represent statistically significant differences as determined in intra-group (Wilcoxon signed-rank test, $\mathrm{p}_{i} 0.05$ ) and inter-group comparisons (Kruskal-Wallis test, $\mathrm{p}_{i} 0.05$ ) with Bonferroni-Holms correction. Also, the force safety margin used to compute the optimal FFE control parameters (dot line, above graph) and the surface registration error threshold (dot lines, graph below) are reported.

tions, whose distortion characteristics resulted inferior.

To comparatively assess the performance of the proposed FFE controller in a controlled and repeatable setup, tests were performed using gelatin phantoms at different concentrations (8$16 \%$ ), where the $8 \%$ concentration gelatin is reported to better represents the viscoelastic properties of the brain. ${ }^{30}$ Strictly dependent on the tool shape and geometry, ${ }^{27}$ the puncturing forces measured during the gelatin characterization protocol resulted consistent to the ranges reported in $[23,24]$, i.e. increasing with respect to gelatin concentration and to the tool insertion velocity. Accordingly, a safety force criterion was considered to evaluate the tissue damage during indentation, depending on the concentration of each gelatin phantom $(0.4 \mathrm{~N}$ with $8 \%, 0.7 \mathrm{~N}$ with $12 \%$, $1 \mathrm{~N}$ with $16 \%$ ). 
The FFE controller proved to significantly reduce the amount of forces applied to the tissue during indentation with respect to both the freehand execution and the robotic assistance without force enhancement, as shown by the equilibrium force index $F_{H}$ (Fig. 9). During FH and RA task executions, the users reach the contact equilibrium using pure visual feedback information, as proven by comparable penetration depths among different concentration gelatin phantoms. Conversely, during FFE task execution, the effect of the haptic feedback provided to the user is shown by comparable tissue interaction forces $\left(F_{H} \approx 0.1 N\right)$ and $50 \%$ reduction of the penetration levels between the $12 \%$ and $16 \%$ gelatin phantoms.Although the median equilibrium forces applied by the users in each experimental condition were below the identified safety levels for each task execution modes and no tissue penetration were registered, the indentation with FFE was significantly reduced. The use of a cooperative robotic assistant was confirmed to allow for a more stable contact with respect to freehand task execution, providing hand tremor rejection due to the reduced bandwidth of the manipulator. As supported by the simulation results, the introduction of the FFE loop did not affect the stability properties of the system during experimental tool-tissue contact, as proved by the comparable and negligible penetration variability results reported in Table 2. The estimate of the hand tremor during contact $(V>0.6 \mathrm{~mm})$ resulted greater than other studies, ${ }^{34}$ but it has to be noted that the users were asked to perform the targeting tasks while standing and without arm support, in order to be comparable to the tasks execution with robotic assistance.

It has to be noted that the experimental validation was performed on static soft tissue phantoms, but in the real surgical scenario predictable and/or unpredictable motions of the organ of interest can occur ${ }^{6,}{ }^{7}$ In this condition, the FFE controller is expected to increase the safety of the surgical procedure, acting as a virtual boundary during unwanted contact and preventing tissue penetration. Tests under dynamic condition would be performed in the future to evaluate this feature. Moreover, the nonlinear characteristic of the FFE controller was optimally tuned based on a predefined safety margin $(50 \%$ of the critical puncturing force), which strictly depends on the mechanical characteristics of the specific tissue, and set constant during the contact. In order to generalize the approach to softer/harder tissues, an adaptive criterion based on the online estimate of the tissue stiffness should be added to the FFE controller.

\section{Conclusion}

Non-linear force feedback torque control was presented and experimentally evaluated on a flexible joint redundant manipulator to provide enhanced haptic perception during cooperatively assisted targeting tasks in surgery. The FFE controller was proven to allow for a tremor-free and safe contact with the tissues, providing 50\% reduction of the indentation level. Future work will address the evaluation of the FFE controller with a pool of expert surgeons on morphologically realistic brain phantom model, in order to exploit the possibility of realizing safety features for cooperatively-controlled interactions with moving organs.

\section{Acknowledgement}

The authors would like to thank Prof. F. Rodriguez y Baena and Prof. D. Prattichizzo for kindly provide the F/T sensors used in the experimental setup. This work was founded by the FP7 ACTIVE project (FP7-ICT-2009-6-270460) and by the Scuola Interpolitecnica di Dottorato.

\section{References}

[1] S. M. Singer and D. L. Akin, A Survey of Quantitative Team Performance Metrics for Human-Robot Collaboration, in Proc. Int. Conf. Environmental Systems, 5248(AIAA) (2011).

[2] E. Beretta, E. De Momi, F. Rodriguez y Baena and G. Ferrigno, Adaptive hands-on control for reaching and targeting tasks in surgery, Int J Adv Robot Syst 12(50) (2015).

[3] A. Szelenyi, L. Bello, H. Duffau, E. Fava, G. C Feigl, M. Galanda, G. Neuloh, F. Signorelli, F. Sala and Workgroup for Intraoperative Management in Low-Grade Glioma Surgery within the European Low-Grade Glioma Network, Intraoperative electrical stimulation in awake craniotomy: methodological aspects of current practice, Neurosurgical focus 28 (2010) E7.

[4] N. Jarrass, J. Paik, V. Pasqui and G. Morel, How can human motion prediction increase transparency?, in Proc. IEEE Int. Conf. Robotics and Automation (2008) pp. 2134-2139.

[5] K. C. Veluvolu and W. T. Ang, Estimation of physiological tremor from accelerometers for real-time applications. Sensors 11(3) (2011) 3020-3036.

[6] C. A. de Oliveira Faria, O. Sadowsky, E. Bicho, G. Ferrigno, L. Joskowiz, M. Shoham, R. Vivanti and E. De Momi, Validation of a stereo camera system to quantify brain deformation due to breathing and pulsatility, Medical Physics, 41(11) (2014).

[7] D. De Lorenzo, E. De Momi, L. Conti, E. Votta, M. Riva, E. Fava, L. Bello and G. Ferrigno, Intraoperative forces and moments analysis on patient head clamp during awake brain surgery, Medical \& Biological Engineering \& Computing 51(3) (2013) 331-341.

[8] A. M. Okamura, Haptic feedback in robot-assisted minimally invasive surgery, Current opinion in urology 19(1) (2009) 102-107.

[9] T. Yamamoto, N. Abolhassani, S. Jung, A. M. Okamura and T. N. Judkins, Augmented reality and haptic interfaces for robotassisted surgery, The International Journal of Medical Robotics and Computer Assisted Surgery 8(1) (2012) 45-56.

[10] D. De Lorenzo, Y. Koseki, E. De Momi, K. Chinzei and A. Okamura, Coaxial needle insertion assistant with enhanced force feedback, IEEE Transactions on Biomedical Engineering 60(2) (2013) 379-389.

[11] M. M. Dalvand, B. Shirinzadeh, S. Nahavandi and J. Smith, Effects of realistic force feedback in a robotic assisted minimally invasive surgery system, Minimally Invasive Therapy \& Allied Technologies 23(3) (2014) 127-135. 
[12] C. R. Wagner and R. D. Howe, Force feedback benefit depends on experience in multiple degree of freedom robotic surgery task, in Robotics, IEEE Transactions 23(6) (2007) $1235-1240$.

[13] B. Demi, T. Ortmaier and U. Seibold, The touch and feel in minimally invasive surgery, Haptic Audio Visual Environments and their Applications, IEEE International Workshop on. (2005).

[14] H. Mayer, I. Nagy, A. Knoll, E. Braun, R. Bauernschmitt and R. Lange, Haptic feedback in a telepresence system for endoscopic heart surgery, in MIT Presence, Teleoperators and Virtual Environments 16(5) (2007) 459-470.

[15] A. Uneri, M. A. Balicki, J. Handa, P. Gehlbach, R. H. Taylor and I. Iordachita, New Steady-Hand Eye Robot with micro-force sensing for vitreoretinal surgery, in Proc. IEEE/RAS-EMBS Int. Conf. on Biomedical Robotics and Biomechatronics (2010) pp. 814-819.

[16] P.J. Berkelman, L. L. Whitcomb, R. H. Taylor and P. Jensen, A miniature instrument tip force sensor for robot/human cooperative microsurgical manipulation with enhanced force feedback, Medical Image Computing and Computer-Assisted InterventionMICCAI (Springer Berlin Heidelberg, 2000) pp. 897-906.

[17] X. He, M. Balicki, P. Gehlbach, J. Handa, R. Taylor and I. Iordachita, A Multi-Function Force Sensing Instrument for Variable Admittance Robot Control in Retinal Microsurgery, in Proc. Int. Conf. Robot Autom (Hongkong, China, 2014) pp. 1411-1418.

[18] D. A. Lawrence, Impedance Control stability properties in common implementation, in Proc. Int. Conf. Robot. Autom. (1988) pp. 1185-1190.

[19] B. Davies, M. Jakopec, S. J. Harris, F. Rodriguez y Baena, A. Barrett, A. Evangelidis, P. Gomes, J. Henckel and J. Cobb, Active-constraint robotics for surgery, in Proceedings of the IEEE 94(9) (2006) 1696-1704.

[20] A. Albu-Schaffer, C. Ott and G. Hirzinger, A passivity based cartesian impedance controller for flexible joint robots - part ii: fullstate feedback, impedance design and experiments, in Proc. Int. Conf. Robot Autom (2004) vol. 3, pp. 2666-2672.

[21] A. M. Okamura, Methods for haptic feedback in teleoperated robotassisted surgery, Int. J Industrial Robot 31(6) (2004) 499-508.

[22] X. He, B. Marcin, P. Gehlbach, J. Handa, R. Taylor and I. Iordachita, A novel dual force sensing instrument with cooperative robotic assistant for vitreoretinal surgery, in Proc. Int. Conf. Robot Autom (2013) pp. 213-218.

[23] K. Miller, C. Kiyoyuki, O. Girma and B. Piotr, Mechanical properties of brain tissue in-vivo: experiment and computer simulation, Journal of Biomechanics 33(11) (2000) 1369-1376.

[24] B. Maurin, L. Barbe, B. Bayle, P. Zanne, J. Gangloff, M. De Mathelin, A. Gangi, L. Soler and A. Forgione, In vivo study of forces during needle insertions, in Proceedings of the medical robotics, navigation and visualisation scientific workshop (2004) pp. 415-422.

[25] P. Schiavone, F. Chassat, T. Boudou, E. Promayon, F. Valdivia and Y. Payan, In vivo measurement of human brain elasticity using a light aspiration device. Medical image analysis 13(4) (2009) 673-678.

[26] P. Marayong, G. D. Hager and A. M. Okamura, Effect of hand dynamics on virtual fixtures for compliant humanmachine interfaces, in Proc. 14th Symposium on Haptic Interfaces for Virtual Environment and Teleoperator Systems (2006) pp. 109-115.

[27] N. Abolhassani, P. Rajni and M. Mehrdad, Needle insertion into soft tissue: A survey Medical Engineering \& Physics 29(4) (2007) 413-431.

[28] D. Stein, H. Monnich, J. Raczkowsky and H. Worn, Visual servoing with an optical tracking system and a lightweight robot for laser osteotomy, in Proc. Int. Conf. Control and Automation (2009) pp. 1896-1900.

[29] R. Horaud and F. Dornaika, Hand-eye calibration, International Journal of Robotics Research 14(3) (1995) 195-210.

[30] D. De Lorenzo, Y. Koseki, E. De Momi, K. Chinzei and A.Okamura, Experimental evaluation of a coaxial needle insertion assistant with enhanced force feedback, in Proc. 33rd Int. Conf. Engineering in Medicine and Biology Society (2011) pp. 3447-3450.

[31] D. Kubus, T. Kroger and F. M. Wahl, On-line rigid object recognition and pose estimation based on inertial parameters, in Proc. Int. Conf. Intelligent Robots and Systems (IROS) (2007) pp. 1402-1408.

[32] F. Janabi-Sharifi, V. Hayward and C. S. J. Chen, Discretetime adaptive windowing for velocity estimation, IEEE Trans. Control Systems Technology 8(6) (2000) 10031009.

[33] S. Holm, A simple sequentially rejective multiple test procedure,Scandinavian journal of statistics (1979) 65-70.

[34] R. A. MacLachlan, B. C. Becker, J. C. Tabares, G. W. Podnar, L. A. Lobes, Jr. and C. N. Riviere, Micron: An actively stabilized handheld tool for microsurgery, IEEE Transactions on Robotics 28(1) (2012) 195-212.

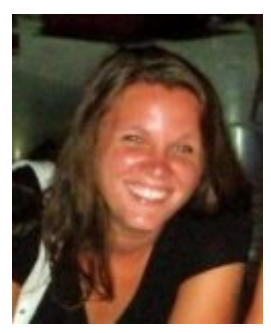

Elisa Beretta graduated cum laude in Biomedical Engineering (2011) and received her $\mathrm{PhD}$ in Bioengineering at Politecnico di Milano (2015) with a thesis entitled Advanced human-robot cooperation in neurosurgery. She is currently works in the Neuroengineering and Medical Robotics laboratory at Politecnico di Milano. Since 2012, she has been involved in the European ACTIVE project and in the framework of Scuola Interpolitecnica di Dottorato. In 2013/14, she was a visiting PhD student at the Mechatronics in Medicine Laboratory of Imperial College, London, working on the development of adaptive control systems for cooperative robotic assistant in neurosurgery. Her research activities cover the fields of computer-assisted and robotic-assisted surgery. 


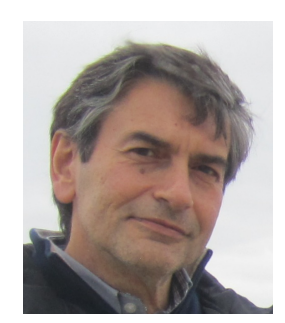

Giancarlo Ferrigno (MSc in Electronic in Engineering in 1983 and $\mathrm{PhD}$ in Bioengineering in 1990), after six years as senior researcher in a private foundation (Pro Juventute Foundation, Milano, from 1984 to 1990), started his academic career at Politecnico di Milano University where he is today Full Professor at the Electronic Information and Bioengineering Department (DEIB) (Assistant Professor in 1990, Associate Professor in 1998 and Full Professor in 2001). From 2004 to 2006 he was appointed head of the Politecnico di Milano PhD School. From 2007 to 2009 he headed the Bioengineering Department. In 2008 he founded the Neuroengineering and Medical Robotics Laboratory. He has taught several courses in the field of artificial intelligence, biosensors, robotics and basic biomedical engineering since 1994. He has carried out scientific activity regarding the application of computer science, system and control engineering and electronic technologies to the study of biological systems, in particular of sensory-motor coordination in man. He is co-author of more than 190 papers (ISI Web of Knowledge), in the movement analysis (pioneer in the field in 1983), technologies for rehabilitation, computational modeling of motor control, Neuroengineering and medical robotics (for surgery and rehabilitation). He is working in the JWG9 ISO standard group for Surgical and rehabilitation Robotics. He has been responsible of many funding grants with companies, Italian Space Agency and European Commission (4th frame program). In the last years he has been the European Coordinator of three FP7 EU projects in the ICT topic. Two of them, ROBOCAST (STREP 2008-2010) and ACTIVE (Integrated project 2011-2015) are in the field of the Surgical and
Computer assisted Robotics. MUNDUS (STREP 2010-2013) is in the field of Assistive and Rehabilitative Robotics

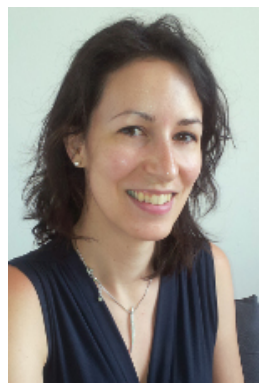

Elena De Momi, PhD in bioengineering (2006) and MSc in biomedical engineering (2002), is an assistant professor at the Department of Electronics, Information and Bioengineering of Politecnico di Milano, Milan, Italy. She was a visiting $\mathrm{PhD}$ student at the Maurice E. Mller Research Center, University of Bern, Switzerland, and at the Institute for Computer Assisted Orthopaedic Surgery Laboratory and Carnegie Mellon University, both in Pittsburgh, Pennsylvania, United States. She is a lecturer of instrumentation and functional evaluation project management in the BSc in biomedical engineering program and on medical robotics in the $\mathrm{PhD}$ in bioengineering program. Since 2006 she has been involved in the NeuroEngineering and Medical Robotics Laboratory and has participated in several national and European grants in the field of computerassisted surgery, keyhole robotic neurosurgery (ROBOCAST), and awake robotic neurosurgery (ACTIVE, project manager). She is a principal investigator of the research network EUROSURGE for partner Politecnico di Milano. De Momi collaborates as an expert with the European Commission in the Seventh Framework Programme. She is part of the editorial board on medical robotics of the International Journal of Advanced Robotic Systems. Her research interests cover the fields of computer-aided surgery, medical robotics, biomechanics, and sensors. 